\title{
Frontier and Closure of a Semi-Pfaffian Set
}

\author{
A. Gabrielov \\ Department of Mathematics, Purdue University, \\ West Lafayette, IN 47907-1395, USA \\ agabriel@math.purdue.edu
}

\begin{abstract}
For a semi-Pfaffian set, i.e., a real semianalytic set defined by equations and inequalities between Pfaffian functions in an open domain $G$, the frontier and closure in $G$ are represented as semi-Pfaffian sets. The complexity of this representation is estimated in terms of the complexity of the original set.
\end{abstract}

\section{Introduction}

The Pfaffian functions introduced by Khovanskii $[\mathrm{K}]$ are analytic functions satisfying a triangular system of Pfaffian differential equations with polynomial coefficients (see Definition 1.1 below). The class of Pfaffian functions includes elementary functions, such as the exponential and logarithmic function, and trigonometric functions in a bounded domain. Many important special functions, such as elliptic integrals, are also Pfaffian.

Khovanskii [K] found an effective estimate for the number of isolated real solutions of a system of equations with Pfaffian functions. This implies global finiteness properties of semi-Pfaffian sets, i.e., semianalytic sets defined by equations and inequalities between Pfaffian functions.

Gabrielov [G1] found a similar estimate for the multiplicity of any complex solution of a system of Pfaffian equations. The latter estimate allowed new finiteness results for the geometry of semi-Pfaffian sets in the real domain, including an effective estimate on the exponent in the Lojasiewicz inequality for semi-Pfaffian functions [G1] and the complexity of a weak stratification of a semi-Pfaffian set [GV], to be derived.

The theory of Pfaffian functions has an important application to computations with usual polynomial functions, based on Khovanskii's theory of "fewnomials." Fewnomials are polynomials containing a limited number of nonzero monomials, of an arbitrarily high degree. Fewnomials can be defined, outside coordinate hyperplanes, as Pfaffian functions of the complexity depending only on the number of nonzero monomials, not on their degrees. This allows the techniques of Pfaffian functions to be applied to 
the complexity of different operations with semialgebraic sets defined by fewnomial expressions in terms of the number of nonzero monomials.

In this paper we apply the finiteness properties established in [G1] to construct a semi-Pfaffian representation for the frontier and closure of a semi-Pfaffian set. Note that the frontier and closure are considered only within the open domain where the Pfaffian functions are defined.

We use a modification of the algorithm suggested in [G3] for the frontier and closure of a semianalytic set. For semi-Pfaffian sets, this allows an effective estimate of the complexity of the semi-Pfaffian representation of the frontier and closure, in terms of the complexity of the original semi-Pfaffian set.

Using the estimates for the multiplicity of a Pfaffian intersection from [G1], we reduce the question whether a given point $x$ belongs to the closure of a semi-Pfaffian set to the question whether $x$ belongs to the closure of an auxiliary semialgebraic set $Z_{x}$, replacing Pfaffian functions by their finite-order Taylor expansions at $x$. We apply algebraic quantifier elimination $[\mathrm{R}],[\mathrm{BPR}]$ to obtain a semialgebraic condition on the coefficients of the polynomials in the formula defining $Z_{x}$, satisfied exactly when $x$ belongs to the closure of $Z_{x}$. As these coefficients are polynomial in $x$ and in the values at $x$ of the original Pfaffian functions and their partial derivatives, the set of those $x$ for which our semialgebraic condition is satisfied is semi-Pfaffian.

The paper is organized as follows. Section 1 introduces Pfaffian functions and semiPfaffian sets. The main result (Theorem 1.1) is formulated at the end of this section. Section 2 presents the necessary finiteness properties of semi-Pfaffian sets, based on the estimate of the multiplicity of Pfaffian intersections from [G1]. Reduction to semialgebraic quantifier elimination and the proof of the main result are given in Section 3. Section 4 contains applications to fewnomial semialgebraic sets.

\section{Pfaffian Functions and Semi-Pfaffian Sets}

Pfaffian functions can be defined in the real or complex domain. We use the notation $\mathbf{K}^{n}$, where $\mathbf{K}$ is either $\mathbf{R}$ or $\mathbf{C}$, in the statements relevant to both cases. Correspondingly, "analytic" means real or complex analytic, and "polynomial" means a polynomial with real or complex coefficients whenever the real or complex domain is considered.

Definition 1.1. A Pfaffian chain of order $r \geq 0$ and degree $\alpha \geq 1$ in an open domain $G \subset \mathbf{K}^{n}$ is a sequence of analytic functions $y_{1}(x), \ldots, y_{r}(x)$ in $G$ satisfying a triangular system of Pfaffian equations

$$
d y_{i}(x)=\sum_{j=1}^{n} P_{i j}\left(x, y_{1}(x), \ldots, y_{i}(x)\right) d x_{j}, \quad \text { for } \quad i=1, \ldots, r .
$$

Here $x=\left(x_{1}, \ldots, x_{n}\right)$, and $P_{i j}\left(x, y_{1}, \ldots, y_{i}\right)$ are polynomials of degree not exceeding $\alpha$. A function $q(x)=Q\left(x, y_{1}(x), \ldots, y_{r}(x)\right)$, where $Q\left(x, y_{1}, \ldots, y_{r}\right)$ is a polynomial of degree not exceeding $\beta>0$, is called a Pfaffian function of degree $\beta$ with the Pfaffian chain $y_{1}(x), \ldots, y_{r}(x)$. Note that the Pfaffian function $q(x)$ is defined only in the open domain $G$ where all the functions $y_{i}(x)$ are analytic, even if $q(x)$ itself can be extended as an analytic function to a larger domain. 
Remark. The above definition of a Pfaffian function corresponds to the definition of a special Pfaffian chain in [G1]. It is more restrictive than the definitions in $[\mathrm{K}]$ and [G1] where Pfaffian chains were defined as sequences of nested integral manifolds of polynomial 1-forms. Both definitions lead to (locally) the same class of Pfaffian functions.

In the following, we fix a Pfaffian chain $y_{1}(x), \ldots, y_{r}(x)$ of degree $\alpha$ defined in an open domain $G \in \mathbf{K}^{n}$, and consider only Pfaffian functions with this particular Pfaffian chain, without explicit reference to the Pfaffian chain, parameters $n, r$, and $\alpha$, and the domain of definition $G$.

For $\mathbf{K}=\mathbf{R}$, we can define a complexification of the Pfaffian chain $y_{1}(x), \ldots, y_{r}(x)$, extending $y_{i}(x)$ as complex-analytic functions into an open domain $\Omega \subset \mathbf{C}^{n}$ containing $G$. Correspondingly, any Pfaffian function can be extended to a complex Pfaffian function in $\Omega$.

We need the following simple properties of Pfaffian functions $[\mathrm{K}],[\mathrm{GV}]$.

Lemma 1.1. The sum (resp. product) of two Pfaffian functions, $p_{1}$ and $p_{2}$ of degrees $\beta_{1}$ and $\beta_{2}$, is a Pfaffian function of degree $\max \left(\beta_{1}, \beta_{2}\right)\left(\right.$ resp. $\left.\beta_{1}+\beta_{2}\right)$.

Lemma 1.2. A partial derivative of a Pfaffian function of degree $\beta$ is a Pfaffian function of degree $\alpha+\beta-1$.

Lemma 1.3. For a Pfaffian function $q(x)=Q\left(x, y_{1}(x), \ldots, y_{r}(x)\right)$ of degree $\beta$, its Taylor expansion

$$
\check{q}_{v}(x, z)=\sum_{k:|k| \leq \nu} \frac{1}{k_{1} ! \cdots k_{n} !} \frac{\partial^{|k|} q}{\partial x^{k}}(z)(x-z)^{k}
$$

of order $v$ at $z \in G$ is a polynomial in $x, z, y_{1}(z), \ldots, y_{r}(z)$ of degree not exceeding $\beta+\alpha v$.

Proof. From Lemma 1.2, the value at $z$ of a partial derivative $\partial^{|k|} q / \partial x^{k}$ is a polynomial in $z$ and $y_{1}(z), \ldots, y_{r}(z)$ of degree at most $\beta+|k|(\alpha-1)$. Hence $\check{q}_{v}(x, z)$ is a polynomial in $x, z, y_{1}(z), \ldots, y_{r}(z)$ of degree at most $\beta+\alpha \nu$.

Definition 1.2. An elementary semi-Pfaffian set in $G \subset \mathbf{R}^{n}$ of the format $(L, n, r, \alpha, \beta)$ is defined by a system of equations and inequalities

$$
x \in G, \quad \operatorname{sign}\left(q_{i}(x)\right)=\sigma_{i}, \quad \text { for } \quad i=1, \ldots, L,
$$

where $\sigma_{i} \in\{-1,0,1\}$ and $q_{i}(x)$ are Pfaffian functions of degree not exceeding $\beta$. A semi-Pfaffian set of the format $(N, L, n, r, \alpha, \beta)$ is a finite union of at most $N$ elementary semi-Pfaffian sets of the format $(L, n, r, \alpha, \beta)$. 
Thus, a semi-Pfaffian set of the format $(N, L, n, r, \alpha, \beta)$ can be defined as

$$
\bigcup_{j=1}^{N} \bigcap_{i=1}^{L}\left\{x \in G, \operatorname{sign}\left(q_{i j}(x)\right)=\sigma_{i j}\right\},
$$

where $q_{i j}(x)$ are Pfaffian functions of degree not exceeding $\beta$ and $\sigma_{i j} \in\{-1,0,1\}$.

Definition 1.3. The closure $\bar{X}$ of a set $X$ in an open domain $G$ is defined as the intersection with $G$ of the usual topological closure of $X$ :

$$
\bar{X}=\{x \in G, \forall \varepsilon>0, \exists z \in X,|x-z|<\varepsilon\} .
$$

The frontier $\partial X$ of $X$ in $G$ is defined as $\partial X=\bar{X} \backslash X$.

Theorem 1.1. Let $X$ be the semi-Pfaffian set (3) in an open domain $G$, and let $(N, L, n$, $r, \alpha, \beta)$ be the format of $X$. Then the frontier $\partial X$ and closure $\bar{X}$ of $X$ in $G$ can be represented as semi-Pfaffian sets, with the format of $\bar{X}$ not exceeding $\left(N^{\prime}, L^{\prime}, n, r, \alpha, \beta^{\prime}\right)$ where

$$
\begin{gathered}
N^{\prime}=N L^{2(n+1)(n+r+1)} d^{(n+r+1) O(n)}, \\
L^{\prime}=L^{2(n+1)} d^{O(n)}, \\
\beta^{\prime}=d^{O(n)} .
\end{gathered}
$$

Here $d=\beta+\alpha(K(n, r, \alpha, \beta)+1)^{2}$, and $K(n, r, \alpha, \beta)$ is as defined in Section 2. The format of $\partial X$ does not exceed $\left(N^{\prime \prime}, L^{\prime \prime}, n, r, \alpha, \beta^{\prime}\right)$ where $N^{\prime \prime}=N^{\prime} L^{N}$ and $L^{\prime \prime}=L^{\prime}+N$.

Remark. It is important that the frontier and closure here are considered within $G$ only. In [G2], we give an example of a semi-Pfaffian set $X \subset G$ such that the closure of $X$ in $\bar{G}$ is not semi-Pfaffian.

\section{Finiteness Properties of Pfaffian Functions}

Definition 2.1. Let $x \in \mathbf{K}^{n}$, and let $f_{1}(x), \ldots, f_{m}(x)$ be analytic functions in an open domain $G$. Let $U$ be an open subset in $G$. The set

$$
X=\left\{x \in U, f_{1}(x)=\cdots=f_{m}(x)=0\right\}
$$

is effectively nonsingular at $z \in G$ if $d f_{1}(x) \wedge \cdots \wedge d f_{m}(x) \neq 0$, for all $x \in X$ close to $z$.

Lemma 2.1. Let $x \in \mathbf{K}^{n}$, and let $f_{1}(x), \ldots, f_{m}(x), s_{1}(x), \ldots, s_{m}(x)$ be analytic functions in an open domain $G \ni 0$. For generic constants $c_{1}, \ldots, c_{m}$, the set

$$
\left\{x, s_{1}(x)=c_{1} f_{1}(x), \ldots, s_{m}(x)=c_{m} f_{m}(x), f_{1}(x) \neq 0, \ldots, f_{m}(x) \neq 0\right\}
$$

is effectively nonsingular at 0 . 
Proof. Consider the mapping $\left(f_{1}, s_{1}\right): \mathbf{K}_{x}^{n} \rightarrow \mathbf{K}^{2}$. Let $\Sigma \subset \mathbf{K}^{n}$ be the germ at 0 of its critical points and let $\Gamma \subset \mathbf{K}^{2}$ be the set of its critical values. Then $\Gamma$ is a germ of a one-dimensional analytic (in the real case, semianalytic) subset in $\mathbf{K}^{2}$. The germ at 0 of the set

$$
Y_{1}=\left\{x, s_{1}(x)=c_{1} f_{1}(x) \neq 0\right\}
$$

is singular when the germ at 0 of $\Gamma \cap\left\{s_{1}=c_{1} f_{1}\right\}$ is one-dimensional. This can happen only for a finite number of values of $c_{1}$. To complete the proof, we can apply the same arguments to the mapping $\left(f_{2}, s_{2}\right): Y_{1} \rightarrow \mathbf{K}^{2}$, and so on.

Definition 2.2. Let $\mathbf{x} \in G \subset \mathbf{K}^{n}$ and $\varepsilon \in \mathbf{K}$. A deformation of a Pfaffian function $q(x)$ is an analytic function $\theta(x, \varepsilon)$ such that $\theta(x, 0)=q(x)$ and, for a fixed $\varepsilon$, the function $\theta(x, \varepsilon)$ is Pfaffian, with the same Pfaffian chain and of the same degree as $q(x)$.

Let $q_{1}(x), \ldots, q_{n}(x)$ be Pfaffian functions. The multiplicity at $z \in G$ of the Pfaffian intersection $q_{1}(x)=\cdots=q_{n}(x)=0$ is defined as the maximal number of isolated complex zeros, for a fixed $\varepsilon \neq 0$, of the system of equations

$$
\theta_{1}(x, \varepsilon)=\cdots=\theta_{n}(x, \varepsilon)=0
$$

converging to $z$ as $\varepsilon \rightarrow 0$. Here $\theta_{i}(x, \varepsilon)$ is any deformation of $q_{i}(x)$, for $i=1, \ldots, n$.

Lemma 2.2. Let $x \in G \subset \mathbf{K}^{n}$, and let $q_{1}(x), \ldots, q_{n}(x)$ be Pfaffian functions of degrees $\beta_{1}, \ldots, \beta_{n}$. Then the multiplicity of the Pfaffian intersection $q_{1}(x)=\cdots=q_{n}(x)=0$ at any point $z \in G$ does not exceed

$$
M\left(n, r, \alpha, \beta_{1}, \ldots, \beta_{n}\right)=2^{r(r-1) / 2} \beta_{1} \cdots \beta_{n}\left[\min (n, r) \alpha+\beta_{1}+\cdots+\beta_{n}-n+1\right]^{r} .
$$

This is a reformulation of Theorem 2.2 from [G1].

The following lemma is a modification of Theorem 3.1 from [G1]. Note that formulas (12) and (13) in [G1] are incorrect: $\alpha+\beta+\gamma$ should be replaced by $2 \alpha+\beta+\gamma$ at both places in both formulas.

Lemma 2.3. Let $x \in G \subset \mathbf{K}^{n}$, and $0 \in G$. Let $q_{1}(x), \ldots, q_{m}(x), f(x)$, and $g(x)$ be Pfaffian functions of degree not exceeding $\beta$. Suppose that $q_{1}(0)=\cdots=q_{m}(0)=$ $f(0)=g(0)=0$. Let $Q=\left\{x \in G, q_{1}(x)=\cdots=q_{m}(x)=0\right\}$. Suppose that $Q^{\prime}=Q \cap\{f g \neq 0\}$ (for $\mathbf{K}=\mathbf{R}, Q^{\prime}=Q \cap\{f>0, g>0\}$ ) is effectively nonsingular at 0 . Let $\Sigma$ be the germ at 0 of the set of critical points of the mapping $\pi=(f, g): Q \rightarrow \mathbf{K}^{2}$, and let $\Gamma$ be a germ at 0 of an irreducible (semi-) analytic curve in $\pi(\Sigma)$, such that $\Gamma^{\prime}=\Gamma \cap \pi\left(Q^{\prime}\right) \neq \emptyset$. Let

$$
K(m, n, r, \alpha, \beta)=M(n, r, \alpha, \underbrace{\beta, \ldots, \beta}_{m+1}, \underbrace{\tau_{m}, \ldots, \tau_{m}}_{n-m-1}),
$$

where $\tau_{m}=(m+2)(\alpha+\beta-1)$ and $M\left(n, r, \alpha, \beta_{1}, \ldots, \beta_{n}\right)$ is as defined in (9). Then, for small $(f, g) \in \Gamma^{\prime}$, we have $f=c g^{\lambda}+o\left(g^{\lambda}\right)$, where $\lambda \leq K(m, n, r, \alpha, \beta)$ is a rational number and $c \in \mathbf{K} \neq 0$. 
Proof. We consider only the complex case. The real case follows easily by complexification.

$\Sigma$ is defined by the Pfaffian equations

$$
q_{1}(x)=\cdots=q_{m}(x)=0, \quad d q_{1}(x) \wedge \cdots \wedge d q_{m}(x) \wedge d f(x) \wedge d g(x)=0 .
$$

The last equation means that all $(m+2)$-minors of

$$
\mathbf{J}=D\left(q_{1}, \ldots, q_{m}, f, g\right) / D\left(x_{1}, \ldots, x_{n}\right)
$$

vanish. According to Lemmas 1.1 and 1.2, these minors are Pfaffian functions of degree not exceeding $\tau_{m}$.

Suppose first that $\Sigma^{\prime}=\Sigma \cap \pi^{-1} \Gamma^{\prime}$ is one-dimensional. There exist linear combinations $h_{1}(x), \ldots, h_{n-m-1}(x)$ of the $(m+2)$-minors of $\mathbf{J}$ such that the germ at 0 of

$$
\left\{h_{1}=\cdots=h_{n-m-1}=0\right\} \cap \pi^{-1} \Gamma^{\prime}
$$

is one-dimensional. If $\Gamma=\left\{f=c g^{\lambda}+o\left(g^{\lambda}\right)\right\}$, where $c \neq 0$, the intersection $\Gamma \cap\{f=\varepsilon\}$ has at least $\lambda$ distinct isolated points converging to the origin as $\varepsilon \rightarrow 0$. This implies that the deformation

$$
q_{1}(x)=\cdots=q_{m}(x)=h_{1}(x)=\cdots=h_{n-m-1}(x)=0, \quad f(x)=\varepsilon
$$

of the Pfaffian intersection

$$
q_{1}(x)=\cdots=q_{m}(x)=h_{1}(x)=\cdots=h_{n-m-1}(x)=0, \quad f(x)=0
$$

has at least $\lambda$ isolated complex zeros $x_{\varepsilon}$ converging to 0 as $\varepsilon \rightarrow 0$. From Lemma 2.2 it follows that $\lambda \leq K(m, n, r, \alpha, \beta)$.

If the dimension of $\Sigma^{\prime}$ is $k>1$, then its intersection with a generic linear $(n-k+1)$ dimensional subspace is nonempty and one-dimensional. In the above arguments we can replace $k-1$ minors by linear functions to obtain an even better estimate for $\lambda$.

Lemma 2.4. Let $x \in G \subset \mathbf{R}^{n}$, and let $f_{1}(x), \ldots, f_{I}(x), g_{1}(x), \ldots, g_{J}(x)$ be Pfaffian functions of degree not exceeding $\beta$. Let

$$
K(n, r, \alpha, \beta)=\max _{0 \leq m<n} K(m, n, r, \alpha, \beta),
$$

where $K(m, n, r, \alpha, \beta)$ is as defined in (10). Let $\varphi(x)=\max _{i}\left|f_{i}(x)\right|$ and $\psi(x)=$ $\min _{j} g_{j}(x)$. Suppose that $0 \in G$ and $\psi(0)=0$. Then, for any real $a>0$ and any integer $v>K(n, r, \alpha, \beta)$, the closure of

$$
S_{a, \nu}=\left\{x \in G, \varphi(x) \leq a \psi(x)^{\nu}, \psi(x)>0\right\}
$$

contains 0 if and only if the closure of $S=\{x \in G, \varphi(x)=0, \psi(x)>0\}$ contains 0. 
Proof. The only nontrivial case is when $\varphi(0)=0$, the closure of $W=\{x \in G, \psi(x)>$ $0\}$ contains 0 , and the closure of $S$ does not contain 0 , i.e., $\varphi(x)>0$ for small $x \in W$. We want to show that the closure of $S_{a, v}$ does not contain 0 in this case.

Let $\delta$ be a positive number such that $G$ contains a cube $C$ of size $\delta$ centered at 0 . Let $V$ be the set where $\varphi(x)$ is minimal over $\{x \in C, \psi(x)=$ const $\}$, i.e.,

$$
V=\left\{x \in C, \varphi(x)=\min _{z \in C, \psi(z)=\psi(x)} \varphi(z)\right\} .
$$

We want to show that there exist a positive constant $c$ and a rational number

$$
\lambda \leq K(n, r, \alpha, \beta)
$$

such that, for small $x \in V$, we have $\varphi(x)>c \psi(x)^{\lambda}$.

Suppose that the closure of $V$ contains 0 . If it does not contain 0 , it should contain another point $x_{0} \in C$ where $\psi\left(x_{0}\right)=0$. We can move the origin from 0 to $x_{0}$ adding, if necessary, linear equations and inequalities when $x_{0}$ is at the boundary $\{|x|=\delta\}$ of $C$.

For two nonempty sets $A \subseteq[1, I]$ and $B \subseteq[1, J]$, let

$$
\begin{aligned}
& W_{A, B}=\left\{x \in W,\left|f_{i}(x)\right|=\varphi(x), \text { for } i \in A,\left|f_{i}(x)\right|<\varphi(x), \text { for } i \notin A,\right. \\
&\left.g_{j}(x)=\psi(x)>0, \text { for } j \in B, g_{j}(x)>\psi(x), \text { for } j \notin B\right\} .
\end{aligned}
$$

We can suppose (multiplying, if necessary, $f_{i}$ and $g_{j}$ by nonzero constants and applying Lemma 2.1) that each set $W_{A, B}$ is effectively nonsingular at 0 , of dimension $n-|A|-$ $|B|+2$.

The set $W$ is a disjoint union of the sets $W_{A, B}$. In particular, there exist $A=\left\{i_{0}, \ldots, i_{k}\right\}$ and $B=\left\{j_{0}, \ldots, j_{l}\right\}$, with $m=k+l<n$, such that the closure of $V \cap W_{A, B}$ contains 0 , and the couple $(A, B)$ is maximal with this property. Changing, if necessary, the signs of $f_{i}$, we can suppose that the closure of $V \cap W_{A, B}^{+}$, where

$$
W_{A, B}^{+}=\left\{x \in W_{A, B}, f_{i}(x)=\varphi(x), \text { for } i \in A\right\},
$$

contains 0 . In this case, $V \cap W_{A, B}^{+}$belongs to the critical set $\Sigma$ of $(f, g): Q \rightarrow \mathbf{R}^{2}$ where $f=f_{i_{0}}, g=g_{j_{0}}$,

$$
Q=\left\{x \in G, f_{i_{0}}(x)=\cdots=f_{i_{k}}(x), g_{j_{0}}(x)=\cdots=g_{j_{l}}(x)\right\},
$$

and $Q^{\prime}=Q \cap\{f>0, g>0\}$ is effectively nonsingular at 0 . The estimate (13) now follows from Lemma 2.3.

Lemma 2.5. Let $x \in G \subset \mathbf{R}^{n}$, and let $f_{1}(x), \ldots, f_{I}(x), g_{1}(x), \ldots, g_{J}(x)$ be Pfaffian functions of degree not exceeding $\beta$. Let $\varphi(x)=\max _{i}\left|f_{i}(x)\right|$ and $\psi(x)=\min _{j} g_{j}(x)$. Let $K(n, r, \alpha, \beta)$ be as defined in (12). Suppose that $0 \in G$ and $\psi(0)=0$. For any positive real numbers $a$ and $b$, and for any integer $v>K(n, r, \alpha, \beta)$ and $\kappa>K(n, r, \alpha, \beta)$, the closure of the set

$$
S_{a, b, v, \kappa}=\left\{x, \varphi(x) \leq a \psi(x)^{\nu}, \psi(x)>b|x|^{\kappa}\right\}
$$

contains 0 if and only if the closure of $S=\{x, \varphi(x)=0, \psi(x)>0\}$ contains 0 . Here $|x|=\max _{i}\left|x_{i}\right|$. 
Proof. According to Lemma 2.4, for any $a \geq 0$ and $v>K(n, r, \alpha, \beta)$, the closure of the set

$$
S_{a, v}=\left\{x, \varphi(x) \leq a \psi(x)^{\nu}, \psi(x)>0\right\}
$$

does not contain 0 when the closure of $S$ does not contain 0 . As $S_{a, b, v, \kappa} \subseteq S_{a, v}$, the closure of $S_{a, b, v, \kappa}$ does not contain 0 in this case.

Suppose now that the closure of $S$ (and the closure of $S_{a, v} \supset S$ ) contains 0 . Let $V$ be the subset of $S_{a, v}$ where $\psi(x)$ is maximal over $|x|=$ const, i.e.,

$$
V=\left\{x \in S_{a, v}, \psi(x)=\max _{z \in S_{a, v},|z|=|x|} \psi(z)\right\} .
$$

The closure of $V$ contains 0 . We want to show that there exist a positive constant $c$ and a rational number

$$
\lambda \leq K(n, r, \alpha, \beta)
$$

such that, for small $x \in V$, we have $\psi(x)>c|x|^{\lambda}$.

Permuting the variables and changing their signs, we can suppose that the closure of $V^{\prime}=V \cap\left\{x_{1}>\max _{i \neq 1}\left|x_{i}\right|\right\}$ contains 0 (if the germ of $V$ at 0 is contained in any of the hyperplanes $\left|x_{i}\right|=\left|x_{j}\right|$, for $i \neq j$, we can find a better estimate for $\kappa$ ).

Let $D=\left\{x \in S_{a, v}, \varphi(x)=a \psi(x)^{\nu}\right\}$. There are two possibilities:

(i) The closure of $V^{\prime} \cap D$ contains 0 .

(ii) The closure of $V^{\prime} \backslash D$ contains 0 , and the closure of $V^{\prime} \cap D$ does not.

We consider here case (i). In case (ii) the arguments are exactly the same as in the proof of Lemma 2.4, with $(\varphi, \psi)$ replaced by $\left(\psi, x_{1}\right)$.

For two nonempty sets $A \subseteq[1, I]$ and $B \subseteq[1, J]$, let

$$
\begin{aligned}
& W_{A, B}=\left\{x,\left|f_{i}(x)\right|=\varphi(x), \text { for } i \in A,\left|f_{i}(x)\right|<\varphi(x), \text { for } i \notin A,\right. \\
&\left.g_{j}(x)=\psi(x)>0, \text { for } j \in B, g_{j}(x)>\psi(x), \text { for } j \notin B\right\} .
\end{aligned}
$$

Following the same arguments as in the proof of Lemma 2.4, we can suppose that each set $W_{A, B}$ is effectively nonsingular at 0 , of dimension $n-|A|-|B|+2$.

Moreover, the sets $Z_{A, B}=W_{A, B} \cap D_{a}$ are effectively nonsingular at 0 . To prove this, let $A=\left\{i_{0}, \ldots, i_{k}\right\}$ and $B=\left\{j_{0}, \ldots, j_{l}\right\}$, and let $f(x)=f_{i_{0}}(x)$ and $g(x)=g_{j_{0}}(x)$. The set $Z_{A, B}$ belongs to the preimage of $\Lambda=\left\{f=a g^{\nu}\right\}$ under the mapping $(f, g): Q \rightarrow \mathbf{R}^{2}$ where

$$
Q=\left\{x, f_{i_{0}}(x)=\cdots=f_{i_{k}}(x), g_{j_{0}}(x)=\cdots=g_{j_{l}}(x)\right\},
$$

and the set of singular points of $Z_{A, B}$ belongs to the preimage of the intersection of $\Lambda$ with the set of critical values $\Gamma$ of $(f, g)$. The germ at 0 of the set of singular points of $Z_{A, B}$ can be nonempty only if $\Lambda$ is a component of the curve $\Gamma$. Due to Lemma 2.3, this is impossible when $v>K(n, r, \alpha, \beta) \geq K(k+l, n, r, \alpha, \beta)$.

The set $D$ is a disjoint union of the sets $Z_{A, B}$. We can choose $A=\left\{i_{0}, \ldots, i_{k}\right\}$ and $B=\left\{j_{0}, \ldots, j_{l}\right\}$ so that the closure of $Z_{A, B}$ contains 0 , and the couple $(A, B)$ is maximal with this property. 
Let $f(x)=f_{i_{0}}(x)$ and $g(x)=g_{j_{0}}(x)$. To prove estimate (15), we want to apply Lemma 2.3 to $g, x_{1}$, and to $m=k+l+1$ functions $q_{1}=f_{i_{1}}-f, \ldots, q_{k}=f_{i_{k}}-f, q_{k+1}=$ $g_{j_{1}}-g, \ldots, q_{m-1}=g_{j_{l}}-g, q_{m}=f-a g^{\nu}$. All these functions are Pfaffian of degree not exceeding $\beta$, except $q_{m}=f-a g^{\nu}$.

To see that the presence of $q_{m}$ does not change the estimate for $\lambda$ in Lemma 2.3, we have to return to the proof of Lemma 2.3 where this estimate was obtained from the estimate for the number of isolated zeros of the Pfaffian deformation (11). This deformation now becomes

$$
q_{1}(x)=\cdots=q_{m}(x)=h_{1}(x)=\cdots=h_{n-m-1}(x)=0, \quad g(x)=\varepsilon,
$$

which is equivalent to

$$
\begin{aligned}
q_{1}(x) & =\cdots=q_{m-1}(x)=h_{1}(x)=\cdots=h_{n-m-1}(x)=0, \\
g(x) & =\varepsilon, \quad f(x)=a \varepsilon^{\nu} .
\end{aligned}
$$

Here $h_{\mu}$ are linear combinations of the coefficients of the $(m+2)$-form

$$
d q_{1} \wedge \cdots \wedge d q_{m} \wedge d g \wedge d x_{1}
$$

However, as $d q_{m} \wedge d g=d f \wedge d g$, this form is equal to

$$
d q_{1} \wedge \cdots \wedge d q_{m-1} \wedge d f \wedge d g \wedge d x_{1}
$$

Thus $h_{1}, \ldots, h_{n-m-1}$ are Pfaffian functions of degree not exceeding $(m+1)(\alpha+\beta-1)<$ $\tau_{m}$. Due to Lemma 2.2, the number of isolated zeros of the deformation (105), converging to 0 as $\varepsilon \rightarrow 0$, does not exceed $K(m, n, r, \alpha, \beta) \leq K(n, r, \alpha, \beta)$.

Lemma 2.6. In the condition of Lemma 2.5, the closure of the set $S$ contains 0 if and only if the closure of the set

$$
T_{a, b, v, \kappa}=\left\{x, \varphi(x) \leq a|x|^{\nu \kappa}, \psi(x)>b|x|^{\kappa}\right\}
$$

contains 0 , for any positive real numbers $a$ and $b$, and for any integer $\nu>K(n, r, \alpha, \beta)$ and $\kappa>K(n, r, \alpha, \beta)$. This is also true without the condition $\psi(0)=0$ of Lemma 2.5.

Proof. Suppose first that $\psi(0)=0$, as in Lemma 2.5. We have $T_{a, b, v, \kappa} \subset S_{a^{\prime}, b, v, \kappa}$ where $a^{\prime}=a / b^{\nu}$. If the closure of $T_{a, b, v, \kappa}$ contains 0 , then the closure of $S_{a^{\prime}, b, \nu, \kappa}$ contains 0 and, due to Lemma 2.5, the closure of $S$ contains 0 . As $\psi(0)=0$, there exists a constant $s>0$ such that $\psi(x) \leq s|x|$, for small $x$. Hence $T_{a, b, v, \kappa} \supset S_{a^{\prime \prime}, b, v \kappa, \kappa}$ where $a^{\prime \prime}=a / s^{\nu \kappa}$. If the closure of $S$ contains 0 , then, due to Lemma 2.5, the closure of $S_{a^{\prime \prime}, b, \nu \kappa, \kappa}$ contains 0 . Hence the closure of $T_{a, b, v, \kappa}$ contains 0 .

If $\psi(0) \neq 0$, then the closure of $S$ contains 0 if and only if $0 \in S$, i.e., if $\psi(0)>0$ and $\varphi(0)=0$. For any positive $a, b, v$, and $\kappa$, the closure of $T_{a, b, v, \kappa}$ contains 0 under exactly the same condition. 


\section{Reduction to the Semialgebraic Case}

Lemma 3.1. Let $x \in \mathbf{R}^{n}$, and let $f_{1}(x), \ldots, f_{I}(x), g_{1}(x), \ldots, g_{J}(x)$ be Pfaffian functions of degree not exceeding $\beta$. Let $K(n, r, \alpha, \beta)$ be as defined in (12). Let $\kappa>$ $K(n, r, \alpha, \beta)$ be an integer number. For $z \in G$, let $F_{i}(x, z)$ be the Taylor expansion of $f_{i}(x)$ at $z$ of order $\kappa^{2}$, and let $G_{j}(x, z)$ be the Taylor expansion of $g_{j}(x)$ at $z$ of order $\kappa$. Then the closure of the semi-Pfaffian set

$$
S=\left\{x, f_{1}(x)=\cdots=f_{I}(x)=0, g_{1}(x)>0, \ldots, g_{J}(x)>0\right\}
$$

contains $z$ if and only if the closure of the following semialgebraic set $S_{z}$ contains $z$ :

$S_{z}=\left\{x, F_{i}(x, z) \leq|x-z|^{\kappa^{2}}\right.$, for $i=1, \ldots, I, G_{j}(x, z)>|x-z|^{\kappa}$, for $\left.j=1, \ldots, J\right\}$.

Proof. According to Lemma 2.6, the closure of $S$ contains $z$ if and only if the closure of the set

$$
T=\left\{x, \varphi(x) \leq a|x-z|^{\kappa^{2}}, \psi(x)>b|x-z|^{\kappa}\right\}
$$

contains $z$, for any positive constants $a$ and $b$. Here $\varphi(x)=\max _{i}\left|f_{i}(x)\right|$ and $\psi(x)=$ $\min _{j} g_{j}(x)$. The latter condition does not depend on the terms of the Taylor expansion at $z$ of $f_{i}(x)$ of order greater than $\kappa^{2}$, and of $g_{j}(x)$ of order greater than $\kappa$. Replacing $f_{i}$ and $g_{j}$ by their Taylor expansions at $z$ of orders $\kappa^{2}$ and $\kappa$, respectively, we can replace $T$ by the semialgebraic set $S_{z}$.

Proof of Theorem 1.1. As the closure of the union equals the union of the closures, it is enough to consider the case of an elementary semi-Pfaffian set:

$$
S=\left\{x \in G, f_{1}(x)=\cdots=f_{I}(x)=0, g_{1}(x)>0, \ldots, g_{J}(x)>0\right\}
$$

with $I+J=L$.

Let $\kappa=K(n, r, \alpha, \beta)+1$. Let $F_{i}(x, z)$ be the Taylor expansion of $f_{i}(x)$ at $z$ of order $\kappa^{2}$ and let $G_{j}(x, z)$ be the Taylor expansion of $g_{j}(x)$ at $z$ of order $\kappa$. According to Lemma 3.1, $\bar{S}$ contains $z \in G$ if and only if $z$ belongs to the closure of the set

$$
\begin{gathered}
S_{z}=\left\{x \in G, F_{i}(x, z) \leq|x-z|^{\kappa^{2}}, \text { for } i=1, \ldots, I,\right. \\
\left.G_{j}(x, z)>|x-z|^{\kappa}, \text { for } j=1, \ldots, J\right\} .
\end{gathered}
$$

Let $y_{1}(x), \ldots, y_{r}(x)$ be the Pfaffian chain for $f_{i}$ and $g_{j}$. According to Lemma 1.3,

$$
F_{i}(x, z)=\Phi_{i}\left(x, z, y_{1}(z), \ldots, y_{r}(z)\right) \quad \text { and } \quad G_{j}(x, z)=\Psi_{j}\left(x, z, y_{1}(z), \ldots, y_{r}(z)\right)
$$

where $\Phi_{i}$ and $\Psi_{j}$ are polynomial in $x, z$, and $y=\left(y_{1}, \ldots, y_{r}\right)$, of degree not exceeding $\beta+\alpha \kappa^{2}$ and $\beta+\alpha \kappa$, respectively.

Thus $z \in \bar{S}$ if and only if the following semialgebraic formula is valid for $z$ and $y_{1}=y_{1}(z), \ldots, y_{r}=y_{r}(z)$ :

$$
\forall \varepsilon>0, \quad \exists x, \quad|x-z| \leq \varepsilon,
$$




$$
\begin{aligned}
& \Phi_{i}(x, z, y) \leq|x-z|^{\kappa^{2}} \text { for } \quad i=1, \ldots, I, \\
& \Psi_{j}(x, z, y)>|x-z|^{\kappa} \text { for } \quad j=1, \ldots, J .
\end{aligned}
$$

This formula contains two blocks of quantifiers, of sizes $k_{1}=1$ and $k_{2}=n$, and $l=n+r$ free variables. It includes $L$ polynomials of degree $d \leq \beta+\alpha \kappa^{2}$.

According to Theorem 1 of [BPR], which is an improvement of Theorem 1.1 of [R], this formula is equivalent to a quantifier-free formula

$$
\bigvee_{i=1}^{N^{\prime}} \bigwedge_{j=1}^{L^{\prime}}\left(\operatorname{sign}\left(Q_{i j}(z, y)\right)=\sigma_{i j}\right)
$$

where $\sigma_{i j} \in\{-1,0,1\}$,

$$
\begin{gathered}
N^{\prime} \leq L^{(l+1)\left(k_{1}+1\right)\left(k_{2}+1\right)} d^{(l+1) O\left(k_{1} k_{2}\right)}=L^{2(n+1)(n+r+1)} d^{(n+r+1) O(n)}, \\
L^{\prime} \leq L^{\left(k_{1}+1\right)\left(k_{2}+1\right)} d^{O\left(k_{1} k_{2}\right)}=L^{2 n+2} d^{O(n)},
\end{gathered}
$$

and $Q_{i j}$ are polynomial in $x, y$ of degree not exceeding

$$
\beta^{\prime}=d^{O\left(k_{1} k_{2}\right)}=d^{O(n)} .
$$

Substituting $y_{k}=y_{k}(z)$ into (19), we obtain a semi-Pfaffian expression for $\bar{X}$ with the properties required in Theorem 1.1 .

The statement for $\partial X=\bar{X} \backslash X$ follows easily from the statement for $\bar{X}$.

\section{Fewnomials}

Definition 4.1. (See $[\mathrm{K}]$ and $[\mathrm{GV}]$.) Let $\mathcal{K}$ be a set of $r$ monomials $u_{1}(x), \ldots, u_{r}(x)$, where $u_{i}(x)=x_{1}^{d_{i 1}} \cdots x_{n}^{d_{i n}}$. A polynomial $P(x)$ is a $\mathcal{K}$-fewnomial of pseudodegree $\beta$ if $P(x)=Q\left(x, u_{1}(x), \ldots, u_{r}(x)\right)$, where $Q$ is a polynomial of degree $\beta$ in $x_{1}, \ldots, x_{n}$, $u_{1}, \ldots, u_{r}$.

Lemma 4.1. Let $\mathcal{K}$ be a set of $r$ monomials in $x=\left(x_{1}, \ldots, x_{n}\right)$, and let $P(x)$ be a $\mathcal{K}$-fewnomial of pseudodegree $\beta$. Then $P(x)$ is a Pfaffian function of degree 1 defined in $G=\left\{x_{1} \cdots x_{n} \neq 0\right\}$, with a Pfaffian chain of rank $n+r$ and degree 2.

Proof. Let $\mathcal{K}=\left\{u_{1}(x), \ldots, u_{r}(x)\right\}$, where $u_{i}(x)=x_{1}^{d_{i 1}} \cdots x_{n}^{d_{i n}}$, and let $P(x)=$ $Q\left(x, u_{1}(x), \ldots, u_{r}(x)\right)$, where $Q$ is a polynomial of degree $\beta$. Let $v_{i}(x)=1 / x_{i}$, for $i=1, \ldots, n$. Then $v_{1}(x), \ldots, v_{n}(x), u_{1}(x), \ldots, u_{r}(x)$ is a Pfaffian chain of rank $n+r$ and degree 2 , as

$$
\begin{gathered}
d v_{i}(x)=-v_{i}(x)^{2} d x_{i} \\
d u_{j}(x)=u_{j}(x)\left(d_{j 1} v_{1}(x) d x_{1}+\cdots+d_{j n} v_{n}(x) d x_{n}\right) .
\end{gathered}
$$


Remark. Note that $G=\left\{x_{1} \cdots x_{n} \neq 0\right\}$ is the domain of definition for a fewnomial $P(x)$ in its Pfaffian representation, due to the presence of $1 / x_{i}$ in the Pfaffian chain, although $P(x)$ itself is defined for all $x$.

Theorem 4.1. Let $\mathcal{K}$ be a set of $r$ monomials in $x=\left(x_{1}, \ldots, x_{n}\right)$, and let $X$ be the semialgebraic set (3), where $G=\left\{x_{1} \neq 0, \ldots, x_{n} \neq 0\right\}$ and all $q_{i j}$ are $\mathcal{K}$-fewnomials of pseudodegree not exceeding $\beta$. Then the closure $\bar{X}$ of $X$ in $G$ is a semialgebraic set

$$
\bigcup_{j=1}^{N^{\prime}} \bigcap_{i=1}^{L^{\prime}}\left\{x \in G, \operatorname{sign}\left(g_{i j}(x)\right)=\sigma_{i j}\right\},
$$

where $\sigma_{i j} \in\{-1,0,1\}$,

$$
\begin{gathered}
N^{\prime}=N L^{2(n+1)(2 n+r+1)} d^{(2 n+r+1) O(n),} \\
L^{\prime}=L^{2(n+1)} d^{O(n),}
\end{gathered}
$$

and all $g_{i j}$ are $\mathcal{K}$-fewnomials of pseudodegree not exceeding $\gamma=(n+1) d^{O(n)}$. Here $d=\beta+2(K(n, n+r, 2, \beta)+1)^{2}$, and $K(n, r, \alpha, \beta)$ is as defined in Section 2. The frontier $\partial X$ of $X$ in $G$ is a semialgebraic set

$$
\bigcup_{j=1}^{N^{\prime \prime}} \bigcap_{i=1}^{L^{\prime \prime}}\left\{x \in G, \operatorname{sign}\left(h_{i j}(x)\right)=\sigma_{i j}\right\},
$$

where $N^{\prime \prime}=N^{\prime} L^{N}, L^{\prime \prime}=L^{\prime}+N$, and all $h_{i j}$ are $\mathcal{K}$-fewnomials of pseudodegree not exceeding $\gamma$.

Proof. Let $\mathcal{K}=\left\{u_{1}(x), \ldots, u_{r}(x)\right\}$. According to Lemma 4.1 and Theorem $1.1, \bar{X} \cap G$ can be represented as a semi-Pfaffian set

$$
\bigcup_{j=1}^{N^{\prime}} \bigcap_{i=1}^{L^{\prime}}\left\{x \in G, \operatorname{sign}\left(s_{i j}(x)\right)=\sigma_{i j}\right\},
$$

where $N^{\prime}$ and $L^{\prime}$ satisfy (5) and (6), with $\alpha=2$ and $r$ replaced by $n+r$, and $s_{i j}$ are Pfaffian functions of degree not exceeding $\beta^{\prime}=d^{O(n)}$, with the Pfaffian chain $1 / x_{1}, \ldots, 1 / x_{n}, u_{1}(x), \ldots, u_{r}(x)$.

As $\beta^{\prime}$ is an even integer number (except for the trivial case $n+r \leq 1$ ) we can replace each $s_{i j}$ in (105) by a $\mathcal{K}$-fewnomial $g_{i j}(x)=\left(x_{1} \cdots x_{n}\right)^{\beta^{\prime}} s_{i j}(x)$ of pseudodegree $\gamma=(n+1) \beta^{\prime}$.

The statement for $\partial X$ follows easily from the statement for $\bar{X}$.

\section{References}

[BPR] S. Basu, R. Pollack, M.-F. Roy, On the combinatorial and algebraic complexity of quantifier elimination, Preprint, 1996. 
[G1] A. Gabrielov, Multiplicities of Pfaffian intersections and the Lojasiewicz inequality. Selecta Math. (N.S.), 1, 113-127 (1995).

[G2] A. Gabrielov, Counter-examples to quantifier elimination for fewnomial and exponential expressions, Preprint, 1995.

[G3] A. Gabrielov, Complements of subanalytic sets and existential formulas for analytic functions, Invent. Math., 125, 1-12 (1996)

[GV] A. Gabrielov and N. Vorobjov, Complexity of stratifications of semi-Pfaffian sets. Discrete Comput. Geom., 14, 71-91 (1995).

[K] A. G. Khovanskii, Fewnomials, AMS Translation of Mathematical Monographs, vol. 88, AMS, Providence, RI, 1991.

[R] J. Renegar, On the computational complexity and geometry of the first order theory of reals, Part III, J. Symbolic Comput., 13, 329-352 (1992).

Received April 2, 1996. 